\title{
High prevalence of human cytomegalovirus in a population of periodontally healthy subjects
}

\author{
Pier-Luigi Foglio-Bonda 1, Mario Gabriele ${ }^{2}$, Filippo Graziani ${ }^{2,3}$, Marco De Andrea ${ }^{4,5}$, Michele Mondini ${ }^{4,5}$, \\ Marisa Gariglio 5
}

\author{
${ }^{1}$ MD, DDS. Dental Clinic, University of Eastern Piedmont “A. Avogadro”, Novara, Italy \\ ${ }^{2}$ MD, DDS. Dept of Surgery, Section of Dentistry and Oral Surgery, University of Pisa, Pisa, Italy \\ ${ }^{3} \mathrm{DMD}, \mathrm{PhD}$. Department of Periodontology, Eastman Dental Institute, University College of London, UK \\ ${ }^{4} \mathrm{PhD}$. Department of Public Health and Microbiology, University of Turin, Turin, Italy \\ ${ }^{5} \mathrm{MD}$, PhD. Department of Clinical and Experimental Medicine, University of Eastern Piedmont “A. Avogadro”, Italy
}

Correspondence:

Clinica Odontostomatologica

Viale Piazza D'Armi 1

28100 Novara. Italy

fbonda@med.unipmn.it

Received: 20/06/2008

Accepted: 12/09/2009
Foglio-Bonda PL, Gabriele M, Graziani F, De Andrea M, Mondini M, Gariglio M. High prevalence of human cytomegalovirus in a population of periodontally healthy subjects. Med Oral Patol Oral Cir Bucal. 2010 Mar 1;15 (2):e292-6.

http://www.medicinaoral.com/medoralfree01/v15i2/medoralv15i2p292.pdf

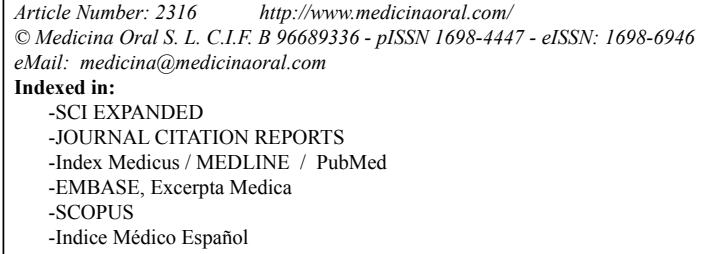

\begin{abstract}
Background: Human cytomegalovirus (HCMV) appears to be more frequent in periodontally affected patients than in healthy control groups. Based on this assumption, it has been suggested that HCMV may play a role in the pathogenesis of periodontal disease. Objective: The objective of this uncontrolled study was to assess the occurrence of HCMV in a large unselected population of periodontally healthy subjects. Study Design: Fifty consecutive periodontally healthy patients satisfied the inclusion criteria. Two samples of gingival crevicular fluids were taken from two non-bleeding on probing sites for each patient. Samples were collected from the anterior and the posterior area. Polymerase chain reaction (PCR) was used to identify the presence of HCMV. Results: HCMV was detected in 17 (33\%) out of 50 participants. Ten subjects showed presence of HCMV on both anterior and posterior sites, whereas the remaining 7 only had HCMV present in the anterior sites. No differences were noticed between HCMV positive and HCMV negative in terms of smoking $(p=0.33)$, drinking habits $(p=0,94)$ or the presence of prosthodontic restorations $(\mathrm{p}=0,89)$. Conclusions: HCMV was detected in a high proportion of periodontally healthy subjects. Its presence was not found to be influenced by smoking or drinking habits.
\end{abstract}

Key words: Periodontal disease, polymerase chain reaction, cytomegalovirus. 


\section{Introduction}

Periodontal diseases are multi-factorial infectious diseases caused, in susceptible individuals, by gram negative bacteria harboured by dental plaque biofilm. Alteration of the balance between bacterial infection and host defences is considered determinant in the development of periodontal diseases (1). Despite pathogenesis of periodontal diseases is srongly related to the presence of periodontal pathogenic bacteria, recent evidence suggests that herpesviruses may also play a significant role in the pathogenesis of severe form of periodontal diseases (2).

Within herpesviruses, human cytomegalovirus HCMV, Epstein-Barr virus (EBV) and herpes virus simplex 1 (HSV-1) seem to take part in the pathogenesis of periodontitis. Indeed, herpesviruses affect periodontal neutrophils function (3). Moreover, a herpesviruses infection of periodontal tissues may determine a shift in the bacterial population of the dental biofilm associated to an elevated prevalence of periodontopathic bacteria such as $\mathrm{P}$. gingivalis, $\mathrm{P}$. intermedia, $\mathrm{T}$. forsythia and $\mathrm{C}$. rectus $(4,5)$. Accordingly, an infection of HCMV and EBV may represent a risk factor for progressive forms of periodontitis as attachment loss may be more likely if HCMV is present (6).

Particularly, HCMV has been involved in various forms of the disease, especially in aggressive, progressive and necrotic periodontal lesions. In aggressive localized periodontitis HCMV appears to increase the odds of having the disease, especially when P. gingivalis is also present (7). Moreover, active sites do show a consistent presence of virus as compared to shallow or stable sites (8). Furthermore, HCMV has been associated with acute necrotizing ulcerative gingivitis and periodontitis in HIV-positive subjects (9).

HCMV is an ubiquitous herpesviruses that infects high percentage of the adult population. HCMV infection is usually asymptomatic in healthy subjects and risks are related to infection in immunocompromized patients. Conflicting data are reported on the occurrence of HCMV at gingival sites. HCMV appears to occur mainly in subjects with periodontitis (10). However, little is known on the occurrence and its impact in periodontally healthy subjects.

This study is a part of a project evaluating the role of herpesviruses on the early stages of periodontal diseases. Aim of the study was to detect the presence of subgingival HCMV in periodontally healthy subjects using polymerase chain reaction technique (PCR).

\section{Material and Methods}

\section{Patient selection}

A group of 131 volunteers was screened for possible inclusion in the study. These subjects were recruited from Italian residents in Novara, Piedmont, Italy. Participants were informed individually, verbally and in writing, about the purpose of the study and signed an informed consent form.

Screening process was performed through a questionnaire and a clinical examination. The questionnaire was used to detect factors or habits that may have an impact on the presence of HCMV in the oral cavity. Particularly, criteria for inclusion were the following: (1) absence of systemic condition, (2) absence of self-reported herpetic infection during the last 6 months, (3) no history of antibiotics within the previous 6 months, (4) no usage of chlorhexidine in the last 6 months, (5) no history of antiviral drugs within the previous 6 months. Moreover, the clinical examiner interviewed each individual as to his/her smoking and drinking habits and previous general health. Subjects who satisfied the inclusion criteria were further examined.

Clinical examination was performed at the Dental Clinic of the University of Eastern Piedmont "A. Avogadro" (Novara, Italy) and it consisted in a dental and oral examination to exclude oro-dental pathologies. Periodontal examination was performed on all teeth and tooth sites, except third molars, were included in the examinations. The following variables were recorded on 6 sites per tooth at the mesio-buccal, buccal, disto-buccall and mesio-lingual, lingual and disto-lingual surfaces of each tooth:

Plaque score: presence/absence of plaque at the cervical part of the tooth scored by running a probe along the tooth surface.

Probing Pocket depth (PPD): measured with a manual $\mathrm{Hu}-$ Friedy PCP15 periodontal probe (Hu-Friedy Inc., Leimen, Germany) to the closest lower millimetre.

Bleeding on Probing (BoP): presence/absence of bleeding within 15 s following pocket probing.

Clinical attachment level (CAL): measured as the distance between cemento-enamel junction and the bottom of the pocket.

Clinical examination was performed by a trained periodontist. Patients to be included had not to show signs of periodontal disease or any visible oral pathology. Furthermore, subjects did not have to show any ortopantomographic evidence of alveolar bone loss.

Routine and specific blood exams were also performed. Particularly, anti-HCMV antibodies were searched. Subjects showing alteration of blood exams were not included.

\section{Sampling}

Two random sites were chosen from each included subjects: one site in the anterior area (incisor, canine and premolars) and one in the posterior area (molars). Sites had not to bleed on probing and not to show CAL loss higher than $3 \mathrm{~mm}$. Samples were collected with hand rolled absorbent paper points (Roeko, Langenau, Germany). Paper points were then suspended in $0.4 \mathrm{ml}$ TE buffer 
(10 nM Tris $\mathrm{HCl} ; 1 \mathrm{nM}$ of EDTA, $\mathrm{pH}$ 7.5) and kept frozen until virologic polymerase chain reaction (PCR) identification. Before PCR reaction, samples were subjected to three cycles of thermic shock $\left(45\right.$ seconds at $+75^{\circ} \mathrm{C}$ followed by $2 \mathrm{~h}$ at $-80^{\circ} \mathrm{C}$ ) in order to induce a mechanical disruption of viral particles and homogenized by vigorous vortex mixing (Reax Top, Heidolph, Germany ).

PCR13.5 $\mu$ l from each sample and $0.25 \mathrm{mM}$ of each primer were used for PCR on a Peltier thermal cycle (PTC-200 MJ Research, Watertown, MA, USA) by using the RED Taq Ready Mix PCR Reaction Mix (Sigma-Aldrich, Milan, Italy) in a total volume of $30 \mu 1$.

DNA amplification was performed with an initial $4 \mathrm{~min}$ incubation at $94^{\circ} \mathrm{C}$, and by 30 cycles using the following thermocycle-step parameters: $94^{\circ} \mathrm{C}$ for $1 \mathrm{~min}$ to denature the DNA, 1 min at $62^{\circ} \mathrm{C}$ for annealing and 2 min at $72^{\circ} \mathrm{C}$, with a final extension of $10 \mathrm{~min}$ at $72^{\circ} \mathrm{C}$. The samples were held at $4^{\circ} \mathrm{C}$ until analyzed. A first round of amplifications was performed using following forward: 5'-CCC GTC GTC GAC GTC GTG ATT-3', and reverse: 5'-GGA AAC ACG AAC GCT GAC GT-3' primers, that amplify the pp65 HCMV gene, which is transcribed late during the infectious cycle. Results were confirmed by a second round of amplification carried out with primers amplifying 392bp from the DNA polymerase viral gene: 5'-CAC GGC CGC CAC CAA GGT-3' and 5'-AGT GGT TGG GCA GGA TAA A-3' respectively for the forward and the reverse. In this case, annealing temperature was decreased to $56^{\circ} \mathrm{C}$.

Positive and negative controls were represented by DNA from infected and not infected leukocytes respectively.

Finally, PCR products were analyzed by electrophoresing $20 \mu \mathrm{l}$ through a $2 \%$ agarose gel (Life Technologies, Gibco BRL, Gaithersburg, MD, USA) with $0.5 \mathrm{mg} / \mathrm{ml}$ of ethidium bromide. Gels were observed and photographed using a UV lamp at 320-nm wavelenght. Low Range MassRuler DNA Ladder (Fermentas Life Science, Vilnius, Lithuania) was used as a molecular size marker.

\section{Statistical analysis and data management}

Continuous data is presented as mean and standard deviations unless stated otherwise. Categorical variables are reported as percentages.

Kolgomorov-Smirnov test was used to ascertain whether data fulfilled normality assumptions. Presence or absence of HCMV, smoking status, drinking habits were defined as a binary variable. Smoking status was recorded also as number of cigarettes and in four categories: non, mild and heavy smoker (more than 10 cigarettes per day). Differences among subgroups according to individuals positivity to HCMV were analysed by Independent t-test or Mann-Whitney U test for continuous data accordingly. Categorical data differences were analyzed by Chi-squared test. The SPSS statistical software package (version 12; SPSS inc. Chicago, IL, USA) was used. Significance was set at $p<0.05$.

\section{Results}

Study population characteristics

A total of 50 individuals (34 women) in the age range 20-59 years (mean: 28 years) were selected. Reasons for exclusion of the remaining 81 are mainly due to previous antibiotic treatment, chlorhexidine usage and presence of systemic diseases.

According to the response, participants were classified in non-smokers, mild smokers and heavy smokers. Smokers were $50 \%$ of the final population. Thirty percent of these group appeared to be heavy smokers.

Occurrence of HCMV

HCMV was detected in 24 samples of the 100 collected. These samples were gathered from 17 subjects $(33 \%$ of the included subjects). Seven subjects showed presence of the virus in both anterior and posterior sites. The remaining 10 subjects had HCMV only in anterior sites (Table 1).

Table 1. Presence of HCMV.

\begin{tabular}{|c|c|c|c|}
\hline & Initials & Age & $\begin{array}{c}\text { Site showing } \\
\text { positivity }\end{array}$ \\
\hline 1 & A.M. & 20 & $3.6-4.4$ \\
\hline 2 & C.C. & 23 & 4.3 \\
\hline 3 & D.M. & 28 & 4.3 \\
\hline 4 & P.G. & 44 & $4.6-4.2$ \\
\hline 5 & J.M. & 22 & 3.3 \\
\hline 6 & E.P. & 24 & 3.3 \\
\hline 7 & D.S. & 27 & 3.3 \\
\hline 8 & A.V. & 24 & 3.3 \\
\hline 9 & D.C. & 40 & $2.5-3.2$ \\
\hline 10 & G.G. & 48 & 3.3 \\
\hline 11 & E.D.A. & 25 & $3.6-3.4$ \\
\hline 12 & M.M. & 24 & $3.6-3.3$ \\
\hline 13 & M.C. & 40 & $3.6-3.4$ \\
\hline 14 & A.S. & 25 & $4.6-3.3$ \\
\hline 15 & E.A. & 35 & 3.2 \\
\hline 16 & L.B. & 31 & 4.2 \\
\hline 17 & V.D. & 22 & 3.3 \\
\hline & & & \\
\hline
\end{tabular}

No differences were gathered among HCMV-positive and HCMV-negative subjects (Table 2). No significant differences (Mann-Whitney, $p=0,38$ ) in terms of age were detected. Subjects negative to HCMV smoked daily 11 cigarettes (DS 6) versus positive subjects who smoked 9 cigarettes (DS 6). This differences was not statistically significant (Indipendent t-test, $\mathrm{p}=0,33$ ). Differences did not reach statistical significance also in any of the categorical data. 
Table 2. Differences between HCMV-positive and HCMV-negative subjects.

\begin{tabular}{|l|c|c|c|}
\hline & HCMV- positive & HCMV - negative & p-values \\
\hline Smokers (\% of the total) & $47 \%$ & $51 \%$ & 0,76 \\
\hline Heavy smokers (\%) & $24 \%$ & $33 \%$ & 0,75 \\
\hline Drinkers (\%) & $35 \%$ & $36 \%$ & 0,94 \\
\hline Prosthodontics (\%) & $35 \%$ & $33 \%$ & 0,89 \\
\hline Cigarettes (N/day) & 9 & 11 & 0,33 \\
\hline Mean Age (years) & 30 & 27 & 0,38 \\
\hline
\end{tabular}

\section{Discussion}

Our data suggest that HCMV can be detected in a significant proportion of consecutive periodontally healthy subjects. A third of our study population showed the presence of HCMV. To our knowledge periodontal investigation on such a vast sample has never been done before. $\mathrm{HCMV}$ is an ubiquitous virus that may be found in large proportion of the adult population. Its vast presence in the population suggests that HCMV may act as a saprofite in the majority of the affected population. Despite the high prevalence, HCMV causes symptoms mainly when alterations of the immune defences are present as in immunocompromised subjects or at an immature stage immune system (11). Most probably, in the majority of the cases HCMV is confined in its latent stage by the immune system. In the last decade, the re-activation of the virus has been supposed to play a role in the development of periodontal diseases $(2,12)$.

This assumption is based on the higher detection of HCMV and herpesviruses in gingival, subgingival tissues, plaque and GCF fluids of periodontally affected subjects rather than healthy ones (13). Nevertheless, periodontal literature did show conflicting data on the occurrence of herpesviruses among periodontally healthy subjects. On one hand, they have not been detected or detected only in a very small proportion of periodontally healthy subjects $(14,15)$.

Our data disagree with these findings. Reasons for disagreement may only be speculated. The available data on periodontally healthy subjects are drawn from a very small number of participants $(6,16)$. Usually, not more than fifteen subjects were enrolled. The authors strongly believe that in detection studies a larger population should be used. Indeed, the theory on the role of herpesviruses in periodontal disease is primarily based on the difference of herpesviruses detection between periodontally affected and non-affected subjects. Interestingly, in our study, involving fifty periodontally healthy subjects, HCMV was detected in a significant portion. Moreover, concerns about the sampling, method and interpretation of previously published data have been raised (13).
Tobacco products are supposed to interact with and reactivate periodontal herpesviruses in an animal model (17). However, this study did not produce convincing evidence that smokers have a higher tendency to be infected by HCMV. On the contrary, although not statistically significant, non infected subjects showed a higher proportion of heavy smokers seemed.

To conclude, HCMV can be frequently detected in periodontally healthy subjects. In our data the frequency of its detection closely resembles previous data on subjects with periodontal disease. Further studies on larger populations are needed in order to evacuate HCMV detection in subjects with periodontal diseases.

\section{References}

1. Madianos PN, Bobetsis YA, Kinane DF. Generation of inflammatory stimuli: how bacteria set up inflammatory responses in the gingiva. J Clin Periodontol. 2005;32 Suppl 6:57-71.

2. Slots J. Herpesviruses in periodontal diseases. Periodontol 2000. 2005;38:33-62.

3. Ongrádi J, Sallay K, Kulcsár G. The decreased antibacterial activity of oral polymorphonuclear leukocytes coincides with the occurrence of virus-carrying oral lymphocytes and epithelial cells. Folia Microbiol (Praha). 1987;32:438-47.

4. Kubar A, Saygun I, Ozdemir A, Yapar M, Slots J. Real-time polymerase chain reaction quantification of human cytomegalovirus and Epstein-Barr virus in periodontal pockets and the adjacent gingiva of periodontitis lesions. J Periodontal Res. 2005;40:97-104. 5. Slots J, Kamma JJ, Sugar C. The herpesvirus-Porphyromonas gingivalis-periodontitis axis. J Periodontal Res. 2003;38:318-23. 6. Kubar A, Saygun I, Yapar M, Ozdemir A, Slots J. Real-time PCR quantification of cytomegalovirus in aggressive periodontitis lesions using TaqMan technology. J Periodontal Res. 2004;39:81-6. 7. Michalowicz BS, Ronderos M, Camara-Silva R, Contreras A, Slots J. Human herpesviruses and Porphyromonas gingivalis are associated with juvenile periodontitis. J Periodontol. 2000;71:981-8. 8. Ting M, Contreras A, Slots J. Herpesvirus in localized juvenile periodontitis. J Periodontal Res. 2000;35:17-25.

9. Contreras A, Mardirossian A, Slots J. Herpesviruses in HIVperiodontitis. J Clin Periodontol. 2001;28:96-102.

10. Contreras A, Slots J. Herpesviruses in human periodontal disease. J Periodontal Res. 2000;35:3-16.

11. Griffiths PD, Walter S. Cytomegalovirus. Curr Opin Infect Dis. 2005;18:241-5

12. Slots J. Update on human cytomegalovirus in destructive periodontal disease. Oral Microbiol Immunol. 2004;19:217-23.

13. Cappuyns I, Gugerli P, Mombelli A. Viruses in periodontal disease - a review. Oral Dis. 2005;11:219-29. 
14. Saygun I, Kubar A, Ozdemir A, Yapar M, Slots J. Herpesviralbacterial interrelationships in aggressive periodontitis. J Periodontal Res. 2004;39:207-12.

15. Contreras A, Nowzari H, Slots J. Herpesviruses in periodontal pocket and gingival tissue specimens. Oral Microbiol Immunol. 2000; $15: 15-8$

16. Konstantinidis A, Sakellari D, Papa A, Antoniadis A. Real-time polymerase chain reaction quantification of Epstein--Barr virus in chronic periodontitis patients. J Periodontal Res. 2005;40:294-8.

17. Park NH, Herbosa EG, Sapp JP. Effect of tar condensate from smoking tobacco and water-extract of snuff on the oral mucosa of mice with latent herpes simplex virus. Arch Oral Biol. 1987;32:4753. 